\title{
Mapping without a map: Exploring the UK business landscape using unsupervised learning
}

\author{
Konstantinos Stathoulopoulos* \& Juan Mateos-Garcia \\ Nesta, 58 Victoria Embankment, London \\ konstantinos.stathoulopoulos@nesta.org.uk
}

\begin{abstract}
Policy interventions have to be timely and tailored to specific sectors of the economic ecosystem to maximise their potential impact. We propose a system based on open data that offers policy makers two capabilities. First, it enables them to explore the digital and tech company space with high granularity through keywords, specific technologies or company names, and identify relevant organisations and those most similar to them. Second, it provides an overview of the ecosystem by creating thematic topics that characterise the activities of these companies. We demonstrate the effectiveness of this system in three activity areas not currently captured in the SIC codes.
\end{abstract}

Keywords - word embeddings;topic modelling;data mining;policy interventions

\section{Introduction}

Growing the digital economy is a major goal for innovation and industrial policy (Borrás \& Edquist, 2013; Aghion \& Besley, 2013). In its Digital Economy Strategy InnovateUK what government agencies need to do to help the sector flourish, including through the provision of infrastructure and equipping individuals with the right skills (InnovateUK, 2015). It also emphasises that an "unprecedented cross-industry collaboration" will be needed "to establish new supply chains and business methods". Therefore, cross-disciplinary, dense networks of companies, universities and experts have to be formed to help ideas spread rapidly through the ecosystem (Rogers, 2003).

\subsection{Limitations of SIC codes}

To design and maximise the effectiveness of interventions, governmental agencies need a detailed view of the components and structure of tech ecosystems. The Standard Industrial Classification (SIC) system used to classify companies into sectors in official statistics, is the main tool for estimating the size and growth of various industries but unfortunately, presents some significant drawbacks.

Companies are classified into a SIC code based on their principal activity, even though organisations usually have cross-sectoral activities. For instance, organisations such as the BBC, might be active in both broadcast and publishing, however these activities are in different SIC codes (Creative Skillset et al., 2013). Research carried out in 2013 suggested that the number of businesses in the information economy and their contribution to the UK economy might be inaccurate if one measures this using SIC codes (Nathan et al., 2014). Moreover, SIC codes in the UK are updated approximately every ten years and therefore, these codes capture a snapshot of the past (in the case of the UK, around the time the iPhone was launched!). This is a particular problem for fast changing creative industries and entrepreneurial ecosystems where startups and SMEs change constantly by adding new products or transforming existing ones. Finally, databases such as Companies House contains information on the majority of UK enterprises, however the lack of qualitative data combined with the lag introduced by industry typologies, 
denies policy makers the ability to understand what these entities are currently working on and provides only a high level view of a sector. Consequently, identifying and comparing companies in a sector using "conventional administrative datasets and industry codes" (Nathan et al., 2014) is difficult and may lead to systematic measurement errors which can distort the true economic output of an industry.

\subsection{Data analytics in policy making}

To help policy makers facilitate collaborations across traditional sector boundaries, we need new ways to analyse the UK business landscape. Open data could provide a way to overcome the limitations of the existing datasets and enrich them with current information. Websites constitute an important medium where organisations promote their products and share their activities. By analysing their content, we can learn more about their domain where a business operates and the technologies it uses.

In this paper, we describe a knowledge representation system that utilises web data to map UK organisations to market sectors. We collect text data from websites, use word embeddings to find the vector representation of words and companies, and topic modelling to create thematic topics using their content. This system provides a flexible way of exploring the UK business landscape both at high and low level. The former representation provides an overview of the economic ecosystem and lets the user examine the sectoral clustering while the latter reveals the similarity between companies as well as between companies and words. As a result, the proposed system can be used as a search engine where the user can query words, or company names to retrieve a list of relevant organisations.

This work is organised as follows: Section $\mathbf{2}$ describes the previous work, while Section 3 shows the data collection pipeline. Section 4 presents how the discussed methods were used and Section 5 shows some results of our work. Section 6 outlines our conclusions and ideas for future work.

\section{Previous Work}

\subsection{Improving SIC codes}

Researchers and organisations have worked with official data to improve the current classification system with Nesta (Bakhshi et al., 2012) measuring the intensity of Creative Industries by reversing the approach that is currently used by the DCMS.

Text data has also been used to improve business classification. Commercial company directories and keyword search were used to create a "Green product" group of organisations (Gök et al., 2014), a code that is missing from the SIC codes. In another project, Shapira et al. (2010) examined the trends in nanotechnology using a similar approach. The size and growth of the Information and Communication Technology (ICT) industries in the UK have also been studied using text data (Nathan et al., 2014). Even though the authors used proprietary data that restricted the transparency of the analysis, their findings strongly suggest that official data underestimate the number of companies in ICT. Furthermore, Mateos-Garcia et al. (2014) used international product directories, scraped text data on games companies and classified them to market sectors. By combining this web dataset with official data, they found that only a third of the games businesses were in the official SIC codes.

We used an NLP approach to overcome the limitations from using proprietary or commercial data and the limiting nature of keywords that require expert knowledge to produce a dictionary to query the data. In particular, topic modelling (Blei, 2012) allows us to find mixture of topics, or market sectors, in company websites. Latent Dirichlet Allocation (Blei et al., 2003) has been used for this purpose and showed compelling results with text of variable length, from tweets (Zhao et al., 2011), to whole articles of academic journals (Blei, 2011). However, LDA cannot capture semantic relations due to its bag-of-words assumption. Word embeddings (Bengio et al., 2003) overcome this limitation by creating the vector representation of words where those that are found in similar context, are also closer in the vector space. Word embeddings were popularised by Mikolov et al. (2013), when they solved the scalability issues and made training on a theoretically infinite amount of text possible. 
Their architecture, word2vec, was extended with Paragraph Vector model (Le \& Mikolov, 2014). Where word2vec creates a dense vector representation of words on sentence level, Paragraph Vector, or doc2vec, can be applied to text of any length. In addition, doc $2 \mathrm{vec}$ receives two inputs, a collection of words and a tag that acts as its unique identifier and can be thought as the topic of the supplied text. Then, it learns the vector representation of both inputs. As a result, word and paragraph vectors are trained simultaneously and exist in the same vector space.

\section{Data Collection}

Since there is not a publicly available directory with UK businesses and their websites, we built a data pipeline that receives a company name and returns a website.

\subsection{Companies House}

Using the data dump of Companies House ${ }^{1}$, we focused on digital and tech SIC codes (Nathan et al., 2014) and created a seed list of organisation names. We examined a specific subset of organisations because although Lai et al. (2015) showed that even though bigger corpora lead to better word vectors, training on domain specific text is of equal importance.

\subsection{Google Custom Search API}

We removed the company type from its registered name and queried the rest on Google Custom Search API. To use the service, we created a search engine that returns only UK results, in English language. This was done to avoid getting non-UK companies with identical names and better Search Engine Optimisation strategy. We retrieved the ten most relevant results and created a rule-based model to find the URL that best matches the queried company name. Specifically, we used Levenshtein distance to measure the dissimilarity between the queried term and the domain name of the URLs in the search results. We selected the URL with the lowest Levenshtein distance and if it was below a predefined threshold, we used Name Entity Recognition on the short description of the specific result from the API with the purpose of identifying the queried company name in there. If there was a match, the website belonged to the company. In the rare cases where more than one URLs passed both tests, we selected the one that ranked higher in the response of the API, since Google Search ranks results based on their relevancy to the query.
In the cases where a match was not found, we deleted the company from the set.

\subsection{Web scraping}

For each company website, we recursively scraped the URLs under the same domain. Specifically, we scraped the homepage and stored the URLs that were in it. Some of these contained only the path to a web page and were fixed by adding the homepage to them. Next, we kept only the URLs within the queried domain and iterated the web scraping for every URL in the stored list. We removed the URLs with a different domain and we ended up with a collection of URLs from the website of the queried company. Finally, for each company, we used the created collection of URLs and scraped their text data.

As a result, we built a dataset with company names, websites, and the text that is contained in every URL of these websites. It should be mentioned we only scraped the text of the 20 shortest URLs to contain the scale of data collection. We scraped the shortest ones because pages such as the homepage, the "About us" and the ones on products and services were assumed to have shorter URLs and provide a better overview of a company's activities.

\section{$4 \quad$ Data Analysis}

\subsection{Text preprocessing}

We aggregated the text from all the URLs of the same company to create a single document with the content of a company website. We tokenized the text and removed any HTML elements, stop-words, rare words and punctuation. We also lemmatised the tokens based on their part-of-speech tag, created bigrams and deleted documents with less than 20 tokens.

\subsection{Training company vectors}

We used doc2vec to find the similarity between UK digital and tech organisations. Doc2vec has been used in sentiment analysis where the tags represented a document's sentiment (Sanguansat, 2016), however in our setting the paragraph tokens were unique and represented company names.

Regarding the hyperparameter optimisation, a large window size was selected since it yields better results (Levy \& Goldberg, 2014) and captures thematic relations 
in the corpus. Moreover, we sub-sampled highly frequent words (Lau \& Baldwin, 2016) and trained the model for 20 epochs.

\subsection{Creating thematic topics}

We transformed the documents bag-of-words and used Latent Dirichlet Allocation to find the mixture of topics contained in them. LDA's hyperparameters were tuned using a grid search and its performance was evaluated with topic coherence (Newman et al., 2010) which measures the interpretability of the resulting topics.

\section{$5 \quad$ Results}

\subsection{Exploratory analysis}

The selected digital and tech SIC codes contain 361,443 companies out of which we used a sample of 64,167 randomly chosen organisations. The rule-based model matched 14,183 companies with websites while web scraping failed to collect data in 320 cases. The absence of SSL certificate and the number of redirections were the main reasons for these failures. When we examined the Local Authorities where companies registered, we found that those with the highest percentage of websites are in central digital hubs and big cities.

\begin{tabular}{|c|c|}
\hline Local Authority District & Companies with website \\
\hline Bristol, City of & $26.1 \%$ \\
\hline Hackney & $25.92 \%$ \\
\hline Sheffield & $25.74 \%$ \\
\hline Southwark & $25.70 \%$ \\
\hline Wiltshire & $25.36 \%$ \\
\hline Brighton and Hove & $25.3 \%$ \\
\hline Richmond upon Thames & $25 \%$ \\
\hline Leeds & $24.91 \%$ \\
\hline Manchester & $24.77 \%$ \\
\hline Barnet & $24.70 \%$ \\
\hline
\end{tabular}

Table 1. Percentage of companies with website in Local Authorities. Only those with more than 250 collected business websites are shown.
Finally, text preprocessing reduced the size of the training set to 12,953 documents with a vocabulary size of 602,367 .

\subsection{Searching the vector space}

We examined the trained word and company vectors by selecting three technologies that are not explicitly included in the SIC codes.

Initially, we queried the trained model using these technologies to semantically related tokens.

\begin{tabular}{|c|c|c|}
\hline blockchain & machine_learning & cyber_security \\
\hline distributed_ledger & artificial_intelligence & cyber \\
\hline ethereum & big_data & security \\
\hline cryptocurrency & algorithms & cyber_threats \\
\hline smart_contracts & data_scientist & cyber_essentials \\
\hline bitcoin & neural_networks & cyber_crime \\
\hline
\end{tabular}

Table 2. Word similarity when using the trained model. Words in bold were used to query it and those below are ordered based on the cosine similarity between them and the query.

We then used the same tokens to search the vector space for companies. It should be mentioned that the five most similar to the queried term companies are shown and all of them are active on the topic they were queried with.

\begin{tabular}{|c|c|c|}
\hline blockchain & machine_learning & cyber_security \\
\hline $\begin{array}{c}\text { APPLIED } \\
\text { BLOCKCHAIN }\end{array}$ & DATRIS & CYBER EYE \\
\hline BLOCKEX & ELLATIVE & $\begin{array}{c}\text { CORDON } \\
\text { SANITAIRE }\end{array}$ \\
\hline COINFIRM & RAPIDMINER & BPH TRAINING \\
\hline IO DIGITAL & MEDIAGAMMA & CSTL \\
\hline $\begin{array}{c}\text { PETERJAMESTH } \\
\text { OMAS.COM }\end{array}$ & BRIGHTERION & SAFONDA \\
\hline
\end{tabular}

Table 3. Similarity between words and paragraph IDs.

The benefits the trained model offers to policy makers are better understood when comparing its results with the SIC codes of these companies. We show (Table 4) that even though companies are working in different domains and using dissimilar technologies, they are 
classified under the same SIC code. In addition, the textual information of these codes does not capture the technologies where these companies specialise:

- 62090: Other information technology service activities

- 62012: Business and domestic software development

- 63990: Other information service activities n.e.c.

- 62020: Information technology consultancy activities

These inefficiencies make the implementation of targeted interventions extremely difficult.

\begin{tabular}{|c|c|c|}
\hline Companies & doc2vec & SIC Code \\
\hline DATRIS & machine_learning & \multirow{6}{*}{62090} \\
\hline ELLATIVE & machine_learning & \\
\hline RAPIDMINER & machine_learning & \\
\hline $\begin{array}{l}\text { CORDON } \\
\text { SANITAIRE }\end{array}$ & cyber_security & \\
\hline COINFIRM & blockchain & \\
\hline IO DIGITAL & blockchain & \\
\hline MEDIAGAMMA & machine_learning & \multirow{3}{*}{62012} \\
\hline BRIGHTERION & machine_learning & \\
\hline BLOCKEX & cyber_security & \\
\hline BPH TRAINING & cyber_security & \multirow{4}{*}{62020} \\
\hline CYBER EYE & cyber_security & \\
\hline SAFONDA & cyber_security & \\
\hline $\begin{array}{c}\text { PETERJAMESTH } \\
\text { OMAS.COM }\end{array}$ & blockchain & \\
\hline $\begin{array}{c}\text { APPLIED } \\
\text { BLOCKCHAIN }\end{array}$ & blockchain & \multirow[t]{2}{*}{63990} \\
\hline CSTL & cyber_security & \\
\hline
\end{tabular}

Table 4. Classification from the trained model in comparison to the SIC code of these companies.

\subsection{Clustering companies}

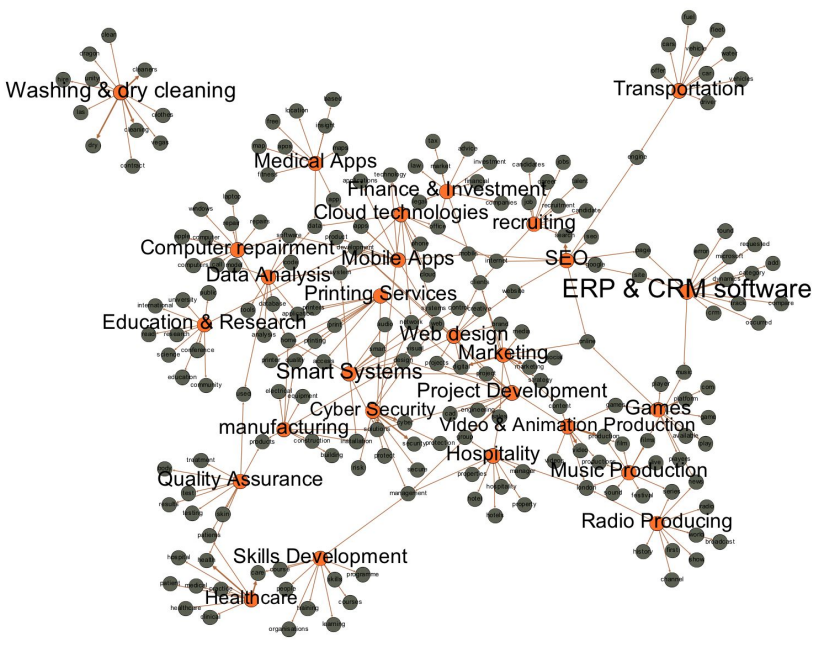

Figure 1. Connection between the identified topics and the top 10 tokens with the highest probability in them. Topic are colour coded with orange and grey nodes are the most probable keywords connected to them.

LDA with 100 topics provided the best results. We identified new topics such as Data Analytics, Smart Systems and Cyber Security while many of the of them were covered by the existing SIC codes. The main advantage of this classification is that the topics, unlike SIC codes, are not mutually exclusive. For instance, a company might be creating Medical Applications but also be in Data Analytics. This would enable the policy makers to find diverse organisations that would assist in establishing cross-disciplinary collaborations.

\section{$6 \quad$ Conclusions \& Future Work}

We present a new approach to find similar companies and classify them to market sectors by using open data and unsupervised learning. Our system provides a way of examining digital and tech businesses on a granular level that overcomes the limitations of SIC codes as well as those that may occur when using non publicly available data.

In future work, we want to replace the rule based model that matches company names to websites with a supervised one in order to make its evaluation easier. Finally, we would like to combine LDA with word embeddings by feeding the former model with the vector representation of words, instead of the one hot encodings (Nguyen et al., 2015). This would enable us to overcome the bog-of-words assumption of LDA and improve the topic coherence by providing information on the semantic structure of the documents. 


\section{References}

Borrás, S., \& Edquist, C. (2013). The choice of innovation policy instruments. Technological forecasting and social change, 80(8), 1513-1522.

Aghion, P., Besley, T., Browne, J., Caselli, F., Lambert, R., Lomax, R., Van Reenen, J. (2013). Investing for Prosperity. Skills, Infrastructure and Innovation, Report of the LSE Growth Commission London: LSE Growth Commission

InnovateUK. (2015) Digital Economy Strategy. [Accessed 02/08/2017]

Rogers, E., M. (2003). Diffusion of Innovations (5th ed).

Bakhshi, H., Freeman, A., \& Higgs, P. L. (2012). A dynamic mapping of the UK's creative industries.

Nathan, M., Rosso, A., Gatten, T., Majmudar, P., \& Mitchell, A. (2013). Measuring the Uk's digital economy with big data. London: NIESR.

Nathan, M., Rosso, A., \& Bouet, F. (2014). Mapping Information Economy Businesses with Big Data: Findings for the UK.

Gök, A., Waterworth, A., \& Shapira, P. (2015). Use of web mining in studying innovation. Scientometrics, 102(1), 653-671.

Mateos-Garcia, J., Bakhshi, H., \& Lenel, M. (2014). A map of the UK games industry. London: Nesta.

Blei, D. M. (2012). Surveying a suite of algorithms that offer a solution to managing large document archives. Communication of the ACM, 55(4), 77-84.

Blei, D. M., Ng, A. Y., \& Jordan, M. I. (2003). Latent dirichlet allocation. Journal of machine Learning research, 3(Jan), 993-1022.

Zhao, W. X., Jiang, J., Weng, J., He, J., Lim, E. P., Yan, H., \& Li, X. (2011, April). Comparing twitter and traditional media using topic models. In European Conference on Information Retrieval (pp. 338-349). Springer, Berlin, Heidelberg.

Blei, D. M. (2012). Probabilistic topic models. Communications of the ACM, 55(4), 77-8

Bengio, Y., Ducharme, R., Vincent, P., \& Jauvin, C. (2003). A neural probabilistic language model. Journal of machine learning research, 3(Feb), 1137-1155.

Mikolov, T., Sutskever, I., Chen, K., Corrado, G. S., \& Dean, J. (2013). Distributed representations of words and phrases and their compositionality. In Advances in neural information processing systems (pp. 3111-3119).

Le, Q., \& Mikolov, T. (2014). Distributed representations of sentences and documents. In Proceedings of the 31st International Conference on Machine Learning (ICML-14) (pp. 1188-1196)

Lai, S., Liu, K., He, S., \& Zhao, J. (2016). How to generate a good word embedding. IEEE Intelligent Systems, 31(6), 5-14.

Sanguansat, P. (2016, February). Paragraph2vec-based sentiment analysis on social media for business in thailand. In Knowledge and Smart Technology (KST), 2016 8th International Conference on (pp. 175-178). IEEE.

Levy, O., \& Goldberg, Y. (2014, June). Dependency-Based Word Embeddings. In ACL (2) (pp. 302-308).

Lau, J. H., \& Baldwin, T. (2016). An empirical evaluation of doc2vec with practical insights into document embedding generation. arXiv preprint arXiv:1607.05368.

Newman, D., Lau, J. H., Grieser, K., \& Baldwin, T. (2010, June). Automatic evaluation of topic coherence. In Human Language Technologies: The 2010 Annual Conference of the North American Chapter of the Association for Computational Linguistics (pp. 100-108). Association for Computational Linguistics.

Nguyen, D. Q., Billingsley, R., Du, L., \& Johnson, M. (2015). Improving topic models with latent feature word representations. Transactions of the Association for Computational Linguistics, 3, 299-313. 